\title{
CHARACTERIZATION OF RHEOLOGICAL PROPERTIES OF SEMISOLID MATERIALS USED AS THERMAL INTERFACES. PART I: A BRIEF REVIEW
}

\author{
FLORINA-CARMEN CIORNEI ${ }^{1 *}$, STELIAN ALACI ${ }^{1}$ \\ I "Stefan cel Mare" University of Suceava, Universitatii St.13, Suceava, 720229, Romania
}

\begin{abstract}
In optimizing the performances of electronic devices, the semisolids used as thermal pastes have a significant part. The interface material should have a good compliance to follow the shape of the contacting surfaces and higher spreadability but also, have increased filler content for high thermal conductivity. The properties of composite materials are theoretically modeled and for designing a semisolid the rheological characterization is required. The paper presents a concise review of the methods used in rheology for characterization of semisolids.
\end{abstract}

Keywords: thermal interface optimization, semisolid, rheology

\section{INTRODUCTION}

Optimization of mechatronic equipment involves problems of electronic miniaturization, integration density and increased power $[1,2]$. These requirements put an emphasis on the heat dissipation problem that is decisive for the performance and reliability of electronic elements. Heat dissipation is mostly achieved by thermal conduction via a heat sink. A better contact between the heat exchanger and the temperature source ensures thus effective cooling, and there are numerous researches for solutions concerning improved thermal contact.

Due to air gaps between the rough mating surfaces, the thermal interface has a resistance that can consume power consistently; thus, reducing air voids is required for reducing the resistance. Different materials like metals, fluids, pastes, particle composites are used as interfaces for reducing junction temperatures. The second task of thermal interface material, besides reducing thermal resistance is maintaining it constant [3].

The properties important in choosing a thermal interface material are [4]: thermal conductivity, thermal resistance, electrical conductivity, phase change temperature, viscosity, operating temperature range, pressure, outgassing, surface finish, ease of application, mechanical properties, long term stability and reliability and cost.

Thermal greases result as a mixture of two constituents, namely a matrix (polymer) and reinforcing particles (filler, ceramic or metallic). The liquid matrix must have thermal stability, wetting features and low modulus of elasticity [4]. Modifying any rheological properties of the constituents, the physical properties of semisolids cam be controlled. Rheology influences increased efficiency in processing and can support designers and users to achieve best possible products [5].

Semisolids fall in the class of non-Newtonian fluids: a well-established relationship between shear stress and shear rate of the material does not exist and the viscosity of the material changes as the shear velocity changes. For a Newtonian fluid, the viscosity is constant as the shear rate varies for a given temperature. The single-point

\footnotetext{
* Corresponding author, email: florina@,fim.usv.ro

(C) 2015 Alma Mater Publishing House
} 
viscosity determinations can be utilized in Newtonian fluids but not in non-Newtonian materials and therefore, rheological measurement techniques for semisolids are important in revealing the flow and deformation behavior.

\section{MATERIALS USED AS THERMAL INTERFACES}

Thermal interface materials are applied between two surfaces with the aim to replace air voids from the thermal interface and to reduce the resistance and, therefore, the junction temperature. These materials must be highly thermally conductive and mate the rough surfaces. They can be thermal pads, films, phase change materials, thermal greases, thermal adhesives, alloy composite materials [3].

There are advantages and drawbacks of each category. Mineral oil used as thermal interface is spreadable and conforms to surfaces, but has a low thermal conductivity [6].

The materials filed with thermally conductive particles have the advantage of a lower cost and workability and by increasing the percentage of particle volume fraction the thermal conductivity can be improved, but this leads to increased viscosity and reduced mating characteristic.

Silicone is widely used as the matrix and particles are, ceramic (alumina, aluminum nitride, zinc oxide, silicon dioxide and beryllium oxides) or metallic (silver, aluminum) [4]. The matrix and filler are mixed and applied to contacting surfaces, pushed to eliminate air from empty spaces. Normally, increasing the particle volume fraction, the thermal conductivity of the thermal grease should also increase. Another aspect concerns optimized particle size distribution that provides a lower viscosity material. In time, thermal grease can dry or move around, these being two main disadvantages. Thermal pastes are widely used due to low costs and efficiency but the workability and mating to surfaces can be further improved. These last requirements can be studied by means of rheology for characterizing, modeling and designing more suitable materials. Semisolid products like pastes are the most difficult to by rheologically characterized because they combine both liquid and solid properties within the same material [5]. These complex dispersions are subjected to complex stresses, specific deformations and shear rates during their lifetime, from their preparation and packaging to their processing, transport, storage and use.

\section{RHEOLOGICAL CHARACTERIZATION OF SEMISOLIDS-MODELS}

Variation in the quality of liquid and semisolid constituents influences the rheological stability and characteristics of semisolids and flow behavior can be an indirect measure of product quality.

Rheological features can be set from flow and deformation reaction of materials when normal and shear stresses are applied [7]. Rheological properties of materials as a function of phase components can be given as functions of viscous, elastic and viscoelastic parameters, using the stress tensor and the strain tensor or shear rate tensor [8]. Viscometric functions are usually employed in stress-shear rate relations for liquids; the elastic functions are used in stress-strain relations for solids and viscoelastic properties refers to a material that presents both viscous and elastic characteristics [9].

One of the factors controlling the flow curve is the amount of material suspended in the liquid, namely the volumic fraction or the phase volume. The phase volume is so important because rheology depends on the hydrodynamic forces acting on the surface of particles [10].

The main classes of fluid flow behavior can be expressed using a basic conventional diagram, shear stresses versus the shear rates. It is possible that flow behavior depends simply on shear rate and not on the period of shear (time-independent), or also depends on the duration of shear (time-dependent). Some categories of timeindependent flow behavior are frequently met, Figure $1[9,11]$.

For Newtonian fluids, there is direct proportionality between the shear rate and shear stress and the graph passes through the origin. For the non-Newtonian fluids, the shear stress versus shear rate graph is not linear, or the plot does not pass through zero, or the material presents time-dependent rheological behavior. 
The shear-thinning fluids are characterized by a curve that passes through the origin of the shear stress-shear rate graph but is convex, that is, an increasing shear rate conducts to a smaller increase in shear stress. Shear-thinning fluids are also called pseudoplastic, but the idiom shear-thinning is chosen for the reason that it is a correct illustration of the stress versus shear rate graph.

Certain materials may not flow until a step stress is surpassed named the yield stress, $\sigma_{0}$. Shear-thinning with yield stress behavior occurs when the shear stress plotted as a function of shear rate gives a straight line presenting a yield stress.

In the case of shear-thickening behavior, the curve passes through the origin of the shear stress-shear rate curve, presents a concavity as an increasing shear stress conducts to a smaller augment in shear rate. The expression dilatant is often utilized for describing shear thickening but since dilatancy indicates raising probe volume, it is not accurate to explain shear-thickening behavior.

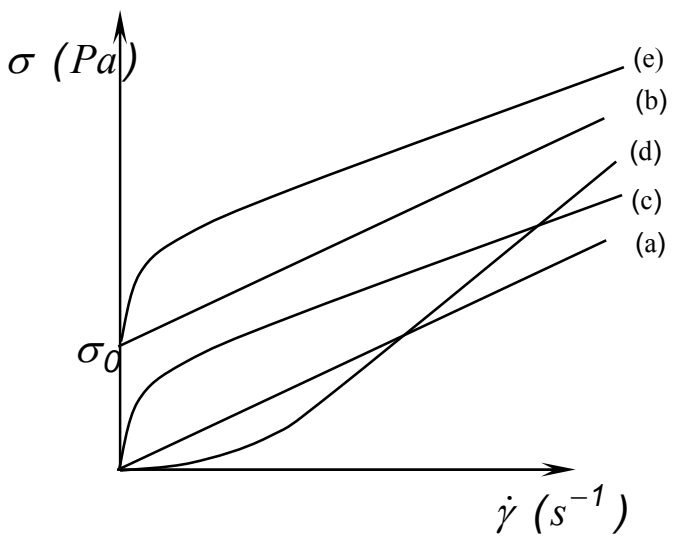

Fig. 1. Time-independent flow types: a) Newtonian; b) plastic (Bingham); c) shear thinning (pseudoplastic); d) shear thickening; e) yield stress plus pseudoplastic (Herschel-Bulkley).

When time-dependent shear thinning occurs in the behavior of a material, it is known as thixotropic. The majority of thixotropic materials are heterogeneous systems, namely they include a dispersed phase. Timedependent shear-thickening behavior is called antithixotropic behavior or rheopectic. When shear rate-shear stress records are obtained by increasing the shear rate and afterwards decreasing it, the two plots will not match and the values of the second are smaller than the first one, the hysteresis loop being thus obtained. For antithixotropic behavior, for decreasing shear rates, the shear stress values are greater than those when rising shear rates.

The zero shear viscosity $\eta_{0}$ is the boundary viscosity at extremely small shear rates. The yield stress $\sigma_{0}$ can be found from the diagram, and the apparent viscosity can be calculated [12] for a specific shear rate (v):

$$
\eta_{a, v}=\sigma_{v} / \dot{\gamma}_{v}
$$

Three types of rheological models are accepted [9]: empirical, theoretical and structural. An empirical model is deduced from evaluation and analysis of experimental results. The theoretical model is consequential to basic notions and offers principles based on the function of structure giving the aspects that control a rheological parameter. The structural model is deduced taking into account the structure and kinetics of changes. Used with experimental data, it can predict values for parameters that characterize the rheological behavior of the probe. Examples of structural models are of Casson that characterizes the materials exhibiting yield stress and that of Cross [9] that describes flow behavior of shear-thinning fluids. The model for a Newtonian fluid is expressed by the relation:

$$
\sigma=\eta \dot{\gamma}
$$


The characterization of a Newtonian fluid states that there is direct proportionality between the shear stress, $\sigma$ and the shear rate, $\dot{\gamma}$ and the parameter, $\eta$, the viscosity, differentiates the experimental values $[8,13]$. For a Bingham plastic fluid, the model must consider a yield stress, $\sigma_{0}$ :

$$
\sigma-\sigma_{0}=\eta^{\prime} \dot{\gamma}
$$

where the Bingham plastic viscosity is denoted by $\eta^{\prime}$. The Newtonian and the Bingham plastic models are mathematically expressed by straight lines shear rate - shear stress, but the first is characterized using one parameter $\eta$ and the second requires two parameters: $\eta^{\prime}$ and $\sigma_{0}$. The shear rate-shear stress values of shearthinning and shear-thickening fluids are models that involve more parameters for depicting the behavior, and the power law representation can be employed. Shear stress-shear rate curves of numerous fluids are linear in double logarithmic coordinate representation, and the power law model illustrates the shear-thinning and shear thickening material manners [7]:

$$
\sigma=K \dot{\gamma}^{n}
$$

where: $K$ is the consistency coefficient, or consistency index, in units of $P a \cdot s^{n}$ and represents the shear stress at a shear rate of $1.0 \mathrm{~s}^{-1} ; n$ is the dimensionless exponent called flow behavior index and relates to Newtonian flow [9].

For the case of a Newtonian fluid $(n=1)$, the consistency index $K$ is, in fact, the viscosity of the fluid, $\eta$. For $n<$ 1 the fluid exhibits shear-thinning behavior and for $n>1$ the fluid shows shear-thickening behavior. Applying logarithms to relation (4), it results:

$$
\log \sigma=\log \mathrm{K}+\mathrm{n} \log \dot{\gamma}
$$

The parameters $K$ and $n$ are found from a graph of $\log \sigma$ function of $\log \dot{\gamma}$. The intercept of the straight line is $\log K$ and $n$ is the slope. It results that the power law model requires two parameters, $K$ and $n$, that can explain shear rate versus shear stress values and is widely used to characterize flows [9]. In almost all identified materials showing shear-thickening, there is a zone of shear-thinning for reduced shear rates.

When yield stress can be evaluated, it can be considered in the power law model and, therefore, the HerschelBulkley model results:

$$
\sigma-\sigma_{O H}=K_{H} \dot{\gamma}^{n H}
$$

where $\dot{\gamma}$ is shear rate $\left(\mathrm{s}^{-1}\right), \sigma$ is shear stress $(\mathrm{Pa}), n_{H}$ is the flow index, $K_{H}$ is the consistency index, and $\sigma_{0 H}$ is yield stress. When the yield stress of a material can be found via another test, $K_{H}$ and $n_{H}$ can be found from the plot in logarithmic coordinates, $\log \left(\sigma-\sigma_{0 H}\right)-\log (\dot{\gamma})$, as the intercept and the slope.

The Casson model is a structure-based model:

$$
\sigma^{0.5}=K_{0 c}+K_{c}(\dot{\gamma})^{0.5}
$$

For a sample behaving following the Casson flow model, the slope $K_{c}$ and intercept $K_{0 c}$ result from a straight line representing the plot of shear rate powered at $0.5,(\dot{\gamma})^{0.5}$ versus shear stress powered at $0.5, \sigma^{0.5}$. The Casson yield stress results finding of the intercept, $\sigma_{0 c}=K_{o c}^{2}$ and the Casson plastic viscosity results from the slope, $\eta_{c a}=\left(K_{c}\right)^{2}$.

Under specified proper settings, all suspensions with high volume fraction of non-aggregating solid fillers will reveal shear-thickening. The particular settings of shear-thickening depend on the phase volume, the particle-size 
distribution and the viscosity of the matrix. The region of shear-thickening usually follows that of the shearthinning. The critical shear rate for shifting to shear-thickening depends slightly on phase volume when the phase volume is near 0.50 . At phase volumes higher than 0.50 , the critical shear rate decreases, even as at phase volumes much below 0.5 , the reverse is valid [10].

\section{EXPERIMENTAL METHODS APPLIED IN RHEOLOGICAL CHARACTERIZATION OF SEMISOLIDS}

Viscosity and rheological tests can be classified into three types [14, 15]:

- Steady shear flow: such as tube and capillary viscometry, falling ball viscometry, and rotational rheometry. The rotational viscometers shear the probe fluid using cylinders, cones or plates. The parallel plate viscometer is widely accepted as an instrument for characterizing the flow behavior of high viscosity fluids.

- Oscillatory tests: where the fluid probe is acted by an oscillating force or deformation. These experiments are made with equipment like oscillatory rheometers or dynamic mechanical testing devices, considering the physical characteristics of the probe.

- Transient condition tests: such as the variation of deformation with time after impulsive modifying the stress or the deviation in stress after a sudden deformation. The scale of the deformation is related to the viscoelastic characteristics of the test probe. The experiments can be performed on devices initially designated to steady shear flow.

Based on these principles, numerous methods were employed [16, 17], but there will be briefly presented only the ones applied in the laboratory.

The systems with coaxial cylinders, with two variants, Searle method, with immobile cup and Couette method, with a passive plunger, are widely used, both for Newtonian and non-Newtonian fluids. These systems are not adequate for semisolids and require a relative large quantity of material to be tested.

The sliding plate rheometer, e.g., employed by [18], Figure 2, with the variant of pack, Figure 3 can be used for experiments with semisolids. The sample to be tested is placed between two plates, a fixed one (2) and a mobile one (1) driven by a force producing a shear stress $\tau=F / A$, where $A$ is the surface on top of which the sample is distributed. The shear strain is $\gamma=x / h$, with $\mathrm{x}$ being the plate displacement and $\mathrm{h}$ the thickness of the tested sample, $\dot{\gamma}=v / h$ is the shear rate and the velocity of the mobile plate is $v=d x / d t$.

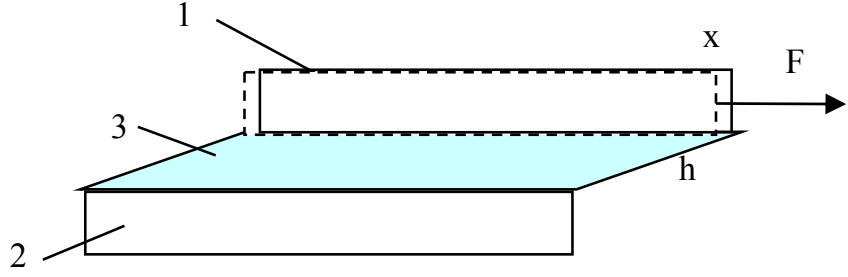

Fig. 2. The sliding plate rheometer.

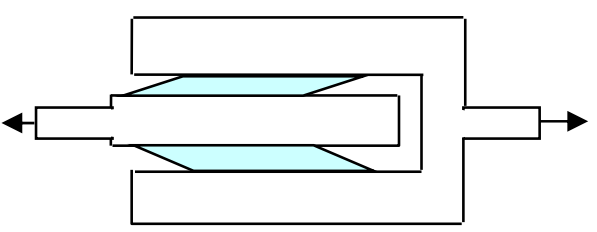

Fig. 3. The sliding pack rheometer.

The sliding pack rheometer is based on the same principle, with two interstitial spaces for shared sample. Knowing the shear stress and shear rate, the apparent viscosity is envisaged.

The filament stretching method was proposed by Matta and Tytus, as Morrison shows [19], the scheme from Figure 4. A semisolid probe (1) is placed between two cylindrical plates (2) and (3). Using an optical device (4), there are measured the length variation and the diameter of the filament that is formed when the inferior plate is suddenly removed by means of a piston system (5)-(6).

Semisolid probes cannot be grabbed at each end of a specimen and stretched, but a variation on this geometry was introduced to measure the elongational viscosity, based on Matta and Tytus [19].This technique stretches a small quantity of polymer solution placed between two round plates. The plates are then separated at a rapid rate, approximating a constant deformation rate. The force on the filament is estimated by a load cell connected to the 
stationary end, and the deformation rate is monitored by video or other optical methods. The change of shape of the boundary offers qualitative information upon the nature of the material, Figure 5 [19].

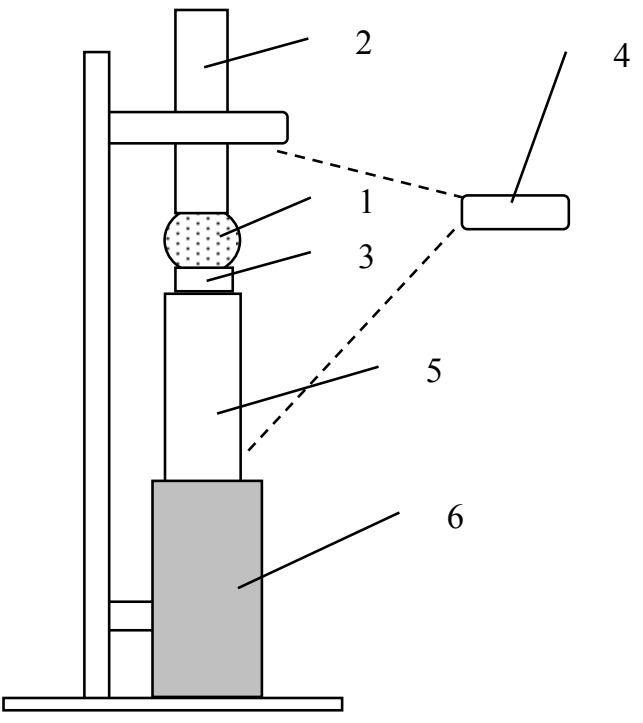

Fig. 4. Filament stretching apparatus scheme.

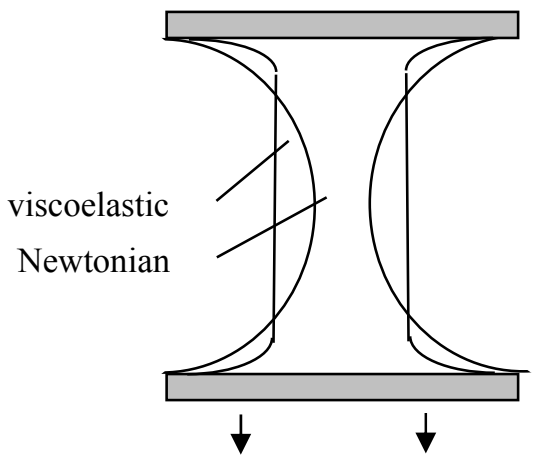

Fig. 5. Filament shape for viscoelastic and Newtonian behavior.

Besides shear stress-shear rate plots, the rheological properties are completed by mechanical properties evaluation, like adherence tests, modulus of elasticity, the working lifetime (defined as the period after which the viscosity increases with $25 \%$ ) and the strength at cyclic thermal fatigue.

\section{CONCLUSIONS}

Thermal pastes are widely used as thermal interface materials due to low cost, workability and controlled thermal conductivity by imposing the percentage of filling particles.

Thermal pastes are composite materials, semisolids resulting from mixing the fluid matrix with solid filler particles. The properties of the liquid phase and solid constituents influence the rheological stability and physical properties of semisolids and flow behavior can be an indirect measure of product quality.

The rheological characterization of a flow is made by representing the shear stress-shear rate dependency; according to the information obtained from the plot, the material can behave Newtonian of non-Newtonian: shear thinning, shear thickening, Bingham or Herschel-Bulkley material. It also can be time dependent or time independent. After qualitative characterization from the shape of the curve, the relations based on a chosen model can give quantitative values for viscosity and yield stress.

The rheometry involves steady shear flow tests, a transient test that can be performed on the same equipment and oscillatory test. For testing semisolids in our laboratory, the sliding plate method is considered most suitable and a second method, filament stretching is envisaged, too.

\section{REFERENCES}

[1] Savija, I., Culham, J.R., Yovanovich, M.M., Marotta, E.E, Review of thermal conductance models for joints incorporating enhancement materials, Journal of Thermophysics and Heat Transfer, vol. 17, no. 1, 2003, p. 4352.

[2] Linderman, R., Brunschwiler, T., Smith, B., Michel, B., High performance thermal interface technology overview, Budapest 17-19 Sept 2007, EDA Publishing Therminic. 
[3] Priyanka, J., Dwivedi, C.K., Thermal interface materials used for improving the efficiency and power handling capability of electronic devices: a review, International Journal Of Innovative Technology \& Creative Engineering, vol. 1, no. 5, 2011, p. 1-9.

[4] Sarvar, F., Whalley, D., Conway, P., Thermal interface materials - a review of the state of the art, IEEE Dresden, 2006, p. 1292-1302.

[5] Jaganath, N., The Application of rheological techniques in the characterization of semisolids in the pharmaceutical industry, dissertation for magister scientiae in the Faculty of Health Sciences at the Nelson Mandela Metropolitan University, University of Port Elizabeth, 2004.

[6] Leong, C.K., Chung, D.D.L, Carbon black dispersions as thermal pastes that surpass solder in providing high thermal contact conductance, Carbon, vol. 41, 2003, p. 2459-2469.

[7] Chhabra, R.P., Bubbles, drops, and particles in non-newtonian fluids, Taylor \& Francis Group, LLC, 2007.

[8] Tudose, R.Z., Volintiru, T., Asandei, N., Lungu, M., Merică, E., Ivan, Gh., Reologia compuşilor macromoleculari, vol III, Reologia stării solide, E.T., București, 1987, p. 236.

[9] Anandha, R.M., Rheology of Fluid, Semisolid and solid foods, principles and applications, Springer Science Business Media, New York, 2014.

[10] Barnes, H.A., Hutton, J.E., Walters, K., An Introduction to rheology, Elsevier Science Publishers B.V., 1993.

[11] Tadro, T.F., Rheology of dispersions, principles and applications, Wiley-VCH Verlag \& Co. KGaA, 2010.

[12] Boger, D.V., Halmos, A.L., Non-newtonian flow i-characterization of fluid behavior, Monash University Clayton, Australia, American Institute of Chemical Engineers, Module C2.1, 1981, p. 1-7.

[13] Jinescu, V.V., Proprietăţile fizice şi termomecanica materialelor plastice, Editura Tehnică București, vol. I, p. 198, vol. II, p. 304, 1979.

[14] Duncan, B.C., Olusanya, A., Review of rheological measurement methods for visco-elastic adhesives, Centre for Materials Measurement and Technology, National Physical Laboratory Teddington, 1999, Report no 1, p. 29.

[15] Yurko, J.A., Fluid Flow Behavior of Semi-solid aluminium at high shear rates, PhD Thesis, MIT, 2001.

[16] Barnes, H.A., A Handbook of Elementary Rheology, Institute of Non-Newtonian Fluid Mechanics University of Wales, 2000.

[17] Shelley, L.A., Gareth, H.M., Duc, A.N., Tam, S., Susan, J.M., Jin, H., David, F.J., An interlaboratory comparison of measurements from filament-stretching rheometers using common test fluids, Journal of Rheology, vol. 45, no. 1, 2001, p. 83-114.

[18] Seung, J.B., Moldenaers, P., Clasen, C., A sliding plate microgap rheometer for the simultaneous measurement of shear stress and first normal stress difference, Review of Scientific Instruments, vol. 82, 2011, p. 1-9.

[19] Morrison, F.A., Understanding rheology, Oxford University Press, 2001. 